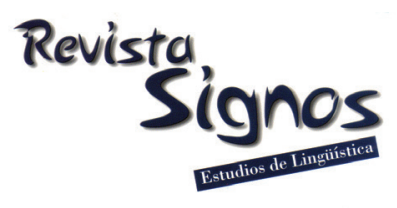

\title{
Uso estratégico de representaciones pictóricas en un texto de ciencias con un detalle seductor en lectores con bajo conocimiento previo
}

\author{
Strategic use of pictorial representations in a Science text with a \\ biographical seductive detail in low prior knowledge readers
}

\section{Gastón Saux \\ CONICET \\ Pontificia Universidad Católica Argentina \\ Argentina \\ gsaux@psi.uba.ar}

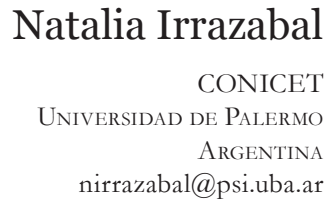

\author{
Débora Burin \\ CONICET \\ Universidad de Buenos Aires \\ ARgentinA \\ dburin@psi.uba.ar
}

Recibido: 12-IV-2013 / Aceptado: 25-V-2015

\section{Resumen}

La Teoría Cognitiva de Aprendizaje Multimedia (Mayer, 2001, 2005) sostiene que el procesamiento conjunto de información lingüística y pictórica resulta en una representación mental integrada del mensaje, y que la adición de representaciones pictóricas puede tanto beneficiar como perjudicar el desempeño asociado a la lectura, dependiendo de la función que cumplan dichos agregados. Este estudio examinó experimentalmente el efecto de asociar representaciones pictóricas con un texto expositivo en castellano (una población sub-representada) que incluyó un dato biográfico tangencial pero interesante (detalle seductor). Ciento cuarenta y siete participantes con bajo conocimiento previo leyeron el texto en una de tres versiones: no ilustrado (condición Solo Detalle Verbal), con una imagen asociada al detalle seductor verbal (condición Multimedia Seductora), o con un diagrama asociado al tema principal del material (condición Multimedia Efectiva). Se registraron indicadores de 
titulación, recuerdo y verificación literal e inferencial de afirmaciones basadas en el texto. Los resultados mostraron que las condiciones multimedia se diferenciaron en sentidos opuestos respecto de la condición de Solo Detalle Verbal. Mientras que la condición Multimedia Seductora presentó el peor desempeño general, la condición Multimedia Efectiva mostró el mejor recuerdo de los contenidos textuales, aunque no se diferenció del grupo de Solo Detalle Verbal en la tarea de verificación inferencial. Estos resultados son interpretados en el marco de la Teoría Cognitiva de Aprendizaje Multimedia.

Palabras Clave: Comprensión, multimedia, texto expositivo, detalle seductor, ilustración.

\begin{abstract}
The Cognitive Theory of Multimedia Learning (Mayer, 2001, 2005) argues that processing joint linguistic and pictorial information results in an integrated mental representation of the message, and that this can both benefit and lessen reading performance, depending on the function of such additions. This study examined the effect of including pictorial representations in an expository text in Spanish that included a tangential but interesting biographical fact (seductive detail). One hundred and forty seven low prior-knowledge participants read the text with the seductive verbal detail in one of three versions: non-illustrated (Verbal Detail Only condition), with an image linked to the verbal seductive detail (Seductive Multimedia condition), or with a diagram linked to the main topic of the text (Effective Multimedia condition). Participants' answers to a title selection task, a free recall task, and a sentence verification task (literal and inferential sentences) were collected. Results showed that the multimedia conditions differed in opposite directions with respect to the Verbal Detail Only condition. Whereas the Seductive Multimedia condition showed the poorest performance, the Effective Multimedia condition showed the best performance in free recall, but did not differ from the Verbal Detail Only condition in the inferential verification task. These results are interpreted in accordance with the Cognitive Theory of Multimedia Learning.
\end{abstract}

Key Words: Comprehension, multimedia, expository text, seductive detail, illustration.

\title{
INTRODUCCIÓN
}

En los últimos veinte años los desarrollos tecnológicos vinculados a la comunicación han modificado considerablemente el acceso a la información. Los espacios tradicionales han sido testigos de la aparición de nuevas formas de transmisión de contenidos que permiten obtener más información en menos tiempo, como enciclopedias online, blogs y artículos breves que buscan interesar al gran público. Estos cambios representan un desafío para el estudio de la comprensión discursiva en contextos aplicados, especialmente el de la enseñanza de las ciencias (Britt, Richter $\&$ Rouet, 2014). Actividades de comprensión frecuentes dentro de estos contextos, 
como determinar el tema principal de un documento, responder preguntas o producir un resumen se ven expuestas a nuevas exigencias estratégicas, vinculadas con el manejo de la abundancia de información. Chung, Oden, Joyner, Sims y Moon (2012), por ejemplo, han reportado recientemente que la probabilidad de hallar información científica errónea o engañosa ha aumentado junto con la necesidad creciente de los sitios formales e informales de publicar nuevos materiales.

En términos cognitivos, los desafíos vinculados con estos cambios en el acceso a la información son complejos y han sido abordados por la investigación desde diversos ángulos. En forma resumida estos abordajes, que en muchos casos se superponen, parten del supuesto de que las explicaciones tradicionales de la comprensión textual (Kintsch, 1998) requieren ser complementadas para dar cuenta de procesos adicionales, no necesariamente descriptos inicialmente por la psicolingüística del texto. Una línea de abordaje se ha interesado notablemente por las actividades de integración mental de múltiples textos, en potencia heterogéneos, para comprender una temática general (Perfetti, Rouet \& Britt, 1999; Rouet \& Britt, 2011). Otra línea ha puesto el foco en los patrones de búsqueda y procesos cognitivos asociados a la navegación de hipertextos (Salmeron, Canas, Kintsch \& Fajardo, 2005; Amadieu \& Salmeron, 2014). Una tercera línea ha puesto el acento en el procesamiento combinado del texto con formatos representacionales no verbales, especialmente el formato pictórico (ilustraciones, gráficos, fotos, etc.), cuya inclusión en los materiales escritos, aunque no es nueva, se ha visto facilitada por los avances tecnológicos (Mayer, 2001; Schnotz, 2005).

Este artículo busca realizar una contribución específica dentro de esta última línea, es decir, el estudio del procesamiento cognitivo asociado al aumento de la cantidad y tipo de información disponible para el lector dentro de un mismo texto. Los modelos cognitivos que explican el procesamiento de mensajes textuales en formato verbal y pictórico, conocidos dentro de la psicología experimental como 'modelos multimedia' (Mayer, 2001, 2005; Schnotz, 2005), han brindado evidencia de que la asimilación de un texto de ciencias con fines educacionales puede tanto mejorar, cuando este incluye representaciones pictóricas asociadas a sus contenidos, como empeorar, si las representaciones pictóricas se asocian con detalles interesantes pero tangencialmente relacionados con el tema principal (Mayer, 2008).

A la fecha, la investigación en el área ha delineado una serie de principios de diseño que buscan promover el procesamiento óptimo de materiales que combinan palabra e imagen en un texto. De acuerdo con el llamado 'principio de coherencia', el material irrelevante debe ser excluido de la presentación multimedia con el objeto de promover la comprensión y el aprendizaje (Mayer, 2005). Sin embargo, esta recomendación puede resultar desafortunada para educadores y autores interesados en aumentar la motivación de los lectores. En otros términos, podar completamente de detalles interesantes a un texto en las situaciones educativas reales podría conducir a un mensaje árido, al tiempo que parece ser más la excepción que la norma (Perales, 2006). 
Las investigaciones que han identificado el mencionado efecto perjudicial de los detalles interesantes pero irrelevantes son numerosas y se extienden a diferentes dominios de las ciencias (Rey, 2012). No obstante, las situaciones de lectura que, manteniendo estos detalles en el texto, buscan hallar condiciones mediadoras o mitigadoras de los mismos han sido menos examinadas. Teniendo esto en cuenta, el propósito general de esta investigación fue estudiar experimentalmente el producto de la lectura de textos de ciencias con información verbal tangencial pero interesante (un detalle seductor) en presentación simple (solo texto) o en dos versiones diferentes de presentación múltiple (texto e imagen). El supuesto fue que la función de uso de la representación pictórica en cada presentación múltiple produciría efectos diferenciables sobre indicadores de recuerdo y comprensión del texto.

\section{Marco teórico}

\subsection{El desafío de comprender textos de ciencias}

La comprensión textual constituye una actividad paulatina y compleja, que requiere del funcionamiento orquestado y en conjunto de múltiples procesos y capacidades. En primer término, todo texto omite información en tanto se asume que el lector podrá agregarla pertinentemente durante la comprensión. Por ello, el lector debe contar con una base previa de conocimientos específicos y generales, apropiada para inferir la información implícita (Kintsch \& van Dijk, 1978). En segundo lugar, el lector debe estar familiarizado con las formas u organizaciones retóricas típicas de los textos (Meyer, 1985). En tercer lugar, el lector debe poner en ejercicio variadas estrategias, orientadas tanto a vincular su punto focal de lectura con otras partes del texto y con su conocimiento previo, como a evaluar y regular la comprensión a intervalos parciales (Garner, 1990; Kintsch, 1998). Finalmente, el lector debe contar con capacidades de memoria operativa, suficientes para realizar las manipulaciones requeridas sobre la información (Just \& Carpenter, 1992). Como resultado, la lectura comprensiva debería producir una representación coherente y jerarquizada de los contenidos del texto, así como un modelo mental de la situación referida en el mismo (van Dijk \& Kintsch, 1983).

En el caso particular del discurso científico, se ha planteado que este presenta atributos distintivos en estructura, contenido y función comunicativa, asociándose con algunos tipos textuales más que con otros, notablemente la exposición y la argumentación (Bassols \& Torrent, 1997; Padilla, Douglas \& López, 2007). Desde una perspectiva psicológica, se asume, por tanto, que los procesos cognitivos involucrados en la comprensión del texto de ciencias presentan particularidades (Escudero \& León, 2007). Al respecto, se ha señalado que estos textos son especialmente difíciles de comprender debido a la complejidad de sus estructuras retóricas, pero también a la baja familiaridad que el lector suele tener con los contenidos (McNamara, 2004; Wiley, Asch, Sanchez \& Jaeger, 2011). El dominio de la física y sus campos afines 
ofrece múltiples ejemplos. Tómese por caso la explicación sobre el funcionamiento de una batería de teléfono portable. Aunque el evento resulte familiar al lector lego, el nivel de análisis científico del mismo (iones con carga eléctrica, reacciones electroquímicas reversibles, etc.) puede paradójicamente resultar poco cercano, dificultando los procesos de integración de la información. Al respecto, Graesser, León y Otero (2002) han propuesto que el texto de física es un caso especialmente exigente para el lector novato a causa del alto grado de abstracción de sus conceptos.

\subsection{El uso de detalles seductores en el texto de ciencias}

Debido a su complejidad, y a pesar de su profusa utilización, los textos de ciencias corren el riesgo de ser percibidos como 'desiertos discursivos' por parte del aprendiz novato, que tiende a ver estos materiales como poco interesantes (Hidi \& Renninger, 2006). Frente a este escenario, los textos pueden incluir anécdotas, episodios personales o datos curiosos con significación emocional. En muchos casos, estos agregados buscan aumentar el atractivo de los textos, en particular cuando se dirigen a lectores que no cuentan con alto nivel de experticia en el tema. Sin embargo, cuando la estrategia consiste en introducir de modo tangencial temas interesantes dentro del texto se ha encontrado que dichos agregados, llamados detalles seductores (DS), tienden a distorsionar o interrumpir los procesos de representación del discurso, dirigiendo las capacidades de quien recibe el mensaje hacia los elementos anecdóticos del texto (Harp \& Mayer, 1997, 1998; Lehman, Schraw, McCrudden \& Hartley, 2007; Mayer, Griffith, Jurkowitz \& Rothman, 2008). Un agregado es considerado DS cuando cumple con tres características: brevedad, alto interés y baja relevancia. La brevedad refiere a la extensión relativa del detalle respecto del resto del texto, que no debería exceder el 30\% del material original (Park, Moreno, Seufert \& Brünken, 2011). El interés refiere a la curiosidad que despierta la información (Harp \& Mayer, 1997). La relevancia refiere al grado de vinculación causal del detalle con el resto del texto (Harp \& Mayer, 1998).

De forma concurrente al estudio de los DS en materiales exclusivamente verbales (Garner, Gillingham \& White, 1989; Rowland, Skinner, Davis-Richards, Saudargas \& Robinson, 2008; McCrudden \& Corkill, 2010), también se ha examinado el impacto de los agregados seductores en presentaciones en formato múltiple, tanto en forma de imágenes (Harp \& Mayer, 1997, 1998; Sánchez \& Wiley, 2006; Park et al., 2011; Sung \& Mayer, 2012; Jaeger \& Wiley, 2014), como sonidos (Moreno \& Mayer, 1999; Harp \& Maslich, 2005) y animaciones (Mayer, Heiser \& Lom, 2001). En líneas generales, estas investigaciones han llegado a conclusiones similares a las obtenidas con DS verbales: el agregado de detalles interesantes pero irrelevantes en múltiples modalidades sensoriales y/o en múltiples formatos representacionales afecta negativamente la representación final del texto.

Ahora bien, la psicología educacional ha propuesto que la inclusión de ilustraciones efectivamente diseñadas suele generar un beneficio sobre las medidas de comprensión 
y aprendizaje del texto de ciencias, en particular en el dominio de la física y ciencias aledañas (Mayer, 2005; Schnotz, 2005). Los datos sugieren que los agregados pictóricográficos pueden, en efecto, actuar como ayudas externas, promoviendo una mejor comprensión del texto. El supuesto es que la imagen posee 'eficiencia computacional' (Gyselinck \& Tardieu, 1999), en tanto representa las relaciones semánticas de modo más sintético y directo que una descripción lingüística. Por ello, siempre que guíen la atención del lector hacia las ideas relevantes, resalten la estructura proposicional o promuevan una activación pertinente de conocimientos, las imágenes resultarían 'efectivas' para el procesamiento del texto (Levin \& Mayer, 1993).

A priori, el planteo de la efectividad de las representaciones pictóricas en los textos educacionales no parece compatibilizar con los estudios sobre detalles seductores, que proponen que las imágenes pueden perjudicar el procesamiento del texto. Esta aparente incompatibilidad ha sido abordada por la Teoría Cognitiva de Aprendizaje Multimedia (Mayer, 2001, 2005) que ha provisto de una explicación del procesamiento conjunto de contenidos verbales y pictóricos de un mensaje, al tiempo que se ha ocupado de dar cuenta del efecto de los DS.

\subsection{Texto y representaciones pictóricas: El procesamiento multimedia}

Desde la lingüística del texto se ha señalado la naturaleza multisemiótica de los textos, o el hecho de que un texto excede el sistema verbal (Kress \& van Leeuwn, 2001). Un texto es concebido como una unidad compleja compuesta por rasgos tanto verbales como no-verbales (rasgos matemáticos, pictóricos, tipográficos, etc.), que el lector debe aprender a utilizar en modo combinado (Parodi, 2010). En sintonía con estos planteos en el nivel descriptivo-formal, la psicolingüística del texto ha señalado un contraste entre los procesos de base del lenguaje y las actividades mentales puestas en juego en la comprensión del discurso o texto. Mientras que "el conocimiento que efectivamente soporta a la función de los procesos de base es estrictamente lingüístico" (Molinari Marotto, 2005: 16), los procesos del nivel textual quedan mejor caracterizados por operaciones no modulares, o que exceden el análisis estrictamente lingüístico de la información. Como proponen De Vega y Cuetos (1999), comprender el significado de un mensaje textual es en cierto modo una actividad extra-verbal, ya que supone activar representaciones conceptuales. Es justamente este carácter de la comprensión textual el que la diferencia de procesos psicolingüísticos primarios, como la categorización de fonemas o el reconocimiento de la forma fonológica de las palabras.

¿Cómo se caracteriza entonces el procesamiento cognitivo de un texto compuesto por rasgos verbales y no-verbales? Dentro de la investigación psicológica, dicha área es conocida como el estudio del procesamiento multimedia, interesada en cómo las personas elaboran y extraen significado a partir de presentaciones en más de un 
formato simbólico (multi-codalidad) y/o en más de una modalidad sensorial (multimodalidad). En este punto es importante señalar una diferencia en el uso del término 'multimodal' entre las teorizaciones lingüística y psicológica. En la investigación lingüística, la multimodalidad refiere a la presencia de diversos modos o recursos en el texto con el propósito de crear significados (Kress \& van Leeuwn, 2001). En este sentido, el uso del término se centra en los aspectos formales del texto o sistemas de representación externos, y puede ser considerado un pre-requisito para pensar en un procesamiento multimedia en el nivel cognitivo. Por otro lado, el uso en psicología del término multimodal refiere a los aspectos mentales o sistemas de representación internos. Dentro de este marco, la multimodalidad se asocia específicamente a una de las formas de procesamiento multimedia, que implica el procesamiento de un mensaje por más de una modalidad sensorial, por ejemplo, un mensaje procesado en simultáneo por los canales sensoriales visual y auditivo (Mayer, 2005). El caso de la multimodalidad sensorial no será examinado en este trabajo. En la presente investigación nos centraremos en el caso de la multicodalidad, es decir, presentaciones que, aunque ocurren en la misma modalidad sensorial (i.e. visión), son procesadas en dos códigos simbólicos diferentes (i.e. el verbal y el viso-espacial), para luego ser integradas en una sola representación semántica del discurso. En concreto, el trabajo estará acotado a textos con representaciones pictóricas denominadas ilustraciones. Este tipo de representaciones no constituyen imágenes aisladas y comprensibles en sí mismas (como una pictografía señalética o una publicidad gráfica), sino que refieren exclusivamente a imágenes que forman parte de un texto (Perales, 2006).

Al respecto, un importante número de investigaciones orientadas al ámbito educacional ha brindado evidencia de que la comprensión y el aprendizaje de textos expositivos mejora cuando se presentan acompañados por imágenes asociadas a los contenidos textuales (Levie \& Lentz, 1982; Mayer, 2009). Dicho efecto ha sido llamado el "efecto facilitador de las ilustraciones" (Gyselinck \& Tardieu, 1999: 195) o 'efecto multimedia' (Mayer, 2001). Dicho efecto ha sido asimilado a nivel teórico en los modelos de procesamiento multimedia que tienen en cuenta tanto el planteo de un doble sistema de representación para la información verbal y no-verbal, como la construcción de representaciones referenciales del texto sobre la base de estas distintas fuentes (para una revisión se sugiere ver Mayer, 2005). El planteo básico de estas propuestas es que las imágenes asociadas con un texto cooperan en la construcción del modelo de situación (van Dijk \& Kintsch, 1983). Ello sería posible porque todo modelo de situación, en tanto representación interna de la situación evocada por el texto, posee un grado de correspondencia con el estado de cosas (y sus relaciones) descriptas, más que con el orden de ingreso de dicha información, constreñido por la naturaleza secuencial de la lectura. Así, dos porciones del texto distantes entre sí pueden estar no obstante fuertemente vinculadas en el modelo, si ello supone una similitud con la situación referida por el texto. La información pictórica, por su parte, también es una representación (externa) que guarda correspondencia con el estado de cosas que busca representar. Entonces, una representación pictórica puede ser vista 
como un medio que captura ciertos aspectos del modelo de situación de modo más sintético y directo que una descripción lingüística. En este sentido, de Vega, Graesser y Glenberg (2008) han propuesto que la imagen formaría parte de los procedimientos que contribuyen a la formación del modelo mental en el curso del procesamiento y, una vez construido el modelo, funcionaría como un instrumento de instanciación del mismo.

Dentro de las propuestas que adhieren a este planteo básico destaca la Teoría Cognitiva del Aprendizaje Multimedia (TCAM) (Mayer, 2001, 2005). De acuerdo con esta teoría, el procesamiento multimedia se inicia con representaciones externas en formato de palabras e imágenes. Luego de estimular los sentidos, estas representaciones externas se codifican y almacenan muy brevemente en la Memoria Sensorial (MS). La MS permite retener temporariamente la información, que es representada en forma de imagen sensorial visual en el caso de las imágenes externas (representaciones pictóricas y palabras escritas) y en forma de imagen sensorial acústica en el caso de las palabras oídas y otros sonidos. A partir de este breve almacenamiento, el procesamiento continúa en la Memoria de Trabajo (MT). Como espacio mental de manipulación activa de la información, la MT recibe las representaciones basadas en modalidad sensorial (visual y auditiva) a las que se esté atendiendo en un cierto momento y las organiza como representaciones o modelos verbales, en el caso de información lingüística, y representaciones o modelos pictóricos, en el caso de información en formato pictórico. Durante la organización, las representaciones verbales pueden activar representaciones no-verbales por asociación y viceversa. Finalmente, los modelos verbal y pictórico son integrados, también en el espacio de la MT, lo cual supone establecer conexiones entre ellos pero también con conocimiento previo almacenado en la MLP, que deberá ser activado para su integración. Este último proceso, la integración, supone la construcción de relaciones entre los modelos verbal y pictórico gracias al establecimiento de correspondencias semánticas (un mapeo mental) de los nodos y relaciones que ambos presentan. Mayer (2001) sostiene que estos procesos no ocurren de modo linear, sino que suponen el uso estratégico de los recursos y el espacio limitado en la MT, pudiendo co-ocurrir, revisarse por monitoreo meta-cognitivo y sobrecargarse. La Figura 1 presenta la propuesta de la TCAM. 


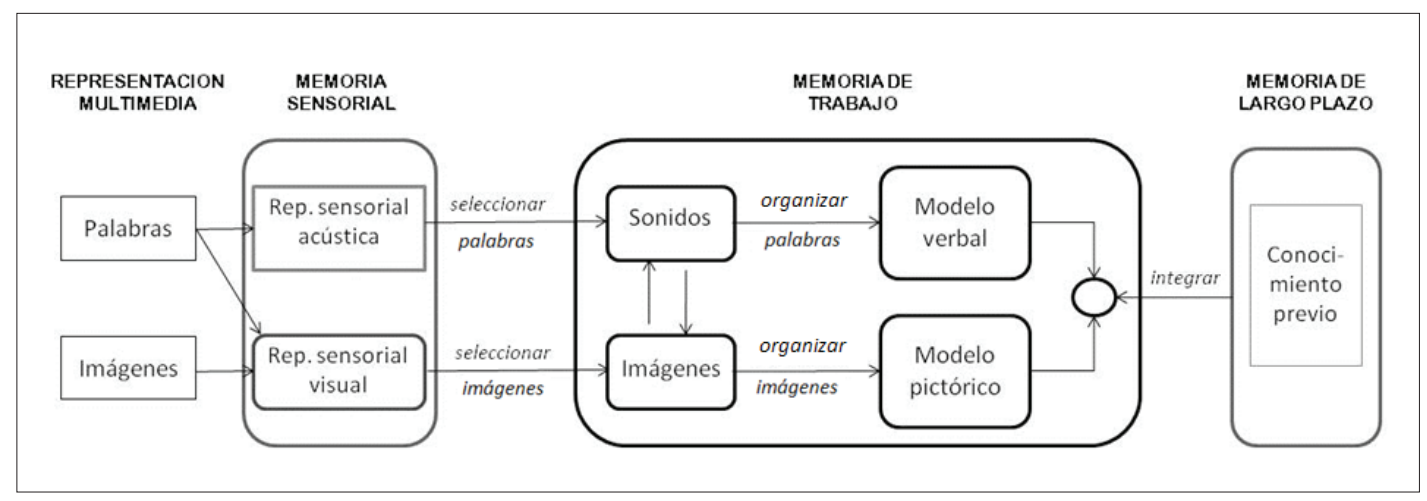

Figura 1. Modelo cognitivo del aprendizaje multimedia (Mayer, 2001).

Bajo esta perspectiva, el efecto de facilitación multimedia ha sido explicado porque las representaciones pictóricas efectivas hacen un buen uso de la eficiencia computacional del formato en imagen (i.e. resaltan viso-espacialmente la organización causal o algún otro aspecto de la exposición escrita) al tiempo que se adecúan a las características del procesamiento cognitivo humano (aprovechan los dos canales de manipulación representacional).

Al mismo tiempo, esta teoría ha explicado el efecto seductor de los detalles al reconocer que los procesos cuentan con capacidades limitadas para su funcionamiento, por lo que el agregado de información tangencial bajo la forma de DS llevaría a una disminución de recursos disponibles para la selección, organización e integración de la información relevante. En concreto, la TCAM ha propuesto dos hipótesis básicas que explican cómo los DS afectan el procesamiento (Mayer, Griffith, Jurkowitz \& Rothman, 2008). La primera hipótesis se centra en los procesos de selección y organización arriba mencionados, y propone que el DS afectaría el funcionamiento de la atención, llevando el foco hacia segmentos poco importantes del material (alteración de la selección), o bien interfiriendo en la conexión de una idea con otra (alteración de la organización). La segunda hipótesis se centra sobre los procesos de integración, que determinan cómo se asimila el mensaje organizado con los conocimientos previos. De acuerdo con esta segunda hipótesis, el DS provoca la activación de esquemas mentales inapropiados en la MLP, favoreciendo la integración del mensaje con conocimientos que se alejan de los contenidos relevantes (alteración de la integración). Como consecuencia, el proceso de representación del texto se 'desviaría', alterando el recuerdo del texto y cualquier actividad que requiera el acceso a la información almacenada a partir del texto.

Atendiendo al potencial beneficio asociado a su procesamiento, podría esperarse que las ilustraciones efectivas ejerzan un contrapeso sobre el efecto perjudicial de los DS. Sin embargo, muchas de las investigaciones en el área multimedia suelen considerar al componente pictórico de la presentación como el DS en sí mismo. Como resultado, tienden a comparar condiciones sin ilustraciones contra condiciones con 
ilustraciones seductoras, o bien contra condiciones con imágenes efectivas (Sánchez \& Wiley, 2006; Sung \& Mayer, 2012; Jaeger \& Wiley, 2014). El impacto de incluir agregados visuales efectivos en textos que mantienen DS verbales ha sido menos examinado (Wade, Schraw, Buxton \& Hayes, 1993; Rowland, Skinner, Skinner, Saudargas, Robinson \& Kirk, 2009).

¿Podría la presencia de una representación pictórica efectiva (i.e. que resalte la organización del texto) reducir los efectos sobre la selección, organización y/o integración del mensaje atribuidos a los DS verbales? Si bien la señalización en el texto de los contenidos esenciales ha sido propuesta por la TCAM como una estrategia a tener en cuenta durante el diseño de presentaciones multimedia (Mayer, 2010), algunos estudios han reportado que las ayudas visuales diseñadas para facilitar la comprensión, tales como el resaltado o la inclusión de cuadros de resumen en el texto, no logran reducir el efecto seductor (Harp \& Mayer, 1998; Rowland et al., 2009; Jaeger \& Wiley, 2014). Las investigaciones asociadas a este problema son todavía escasas, más aún dentro de los estudios experimentales en lengua castellana.

\section{Marco metodológico}

Esta investigación se propuso: (a) estudiar experimentalmente la comprensión y el recuerdo de textos de ciencias con información tangencial pero interesante (un DS) en presentación simple (solo texto) o múltiple (texto e imagen) en estudiantes universitarios con bajo conocimiento previo sobre el tema; (b) examinar los efectos facilitador y seductor en materiales en castellano, comparando la presentación del texto en versión exclusivamente verbal y en dos versiones ilustradas; y (c) examinar la capacidad de una ilustración efectiva para mitigar el efecto seductor.

Se recolectaron tres indicadores. Siguiendo la tradición de los estudios sobre detalles seductores (Mayer, 2001) se utilizó una tarea de recuerdo para examinar la representación en la memoria luego de la lectura. Adicionalmente, se utilizaron dos tareas clásicas de comprensión: una tarea de titulación y una tarea de respuesta a preguntas con opción múltiple (con y sin disponibilidad de los materiales) para examinar el grado de integración de la representación textual.

\subsection{Hipótesis de estudio}

Sobre la base de los objetivos, se plantearon las siguientes hipótesis experimentales:

Hipótesis general: la función de uso de la representación pictórica producirá efectos sobre la representación mental de un texto expositivo con un DS verbal biográfico.

Hipótesis específica 1: la inclusión de una representación pictórica asociada al DS verbal llevará a un desempeño significativamente más bajo en tareas que evalúen la representación textual resultante, en comparación con la lectura del mismo texto sin agregado pictórico. 
Hipótesis específica 2: la inclusión de una representación pictórica efectiva, asociada a la explicación presentada en el texto, reducirá el efecto seductor del detalle verbal, observándose un desempeño significativamente más alto en tareas que evalúen la representación textual resultante, en comparación con la lectura del mismo texto sin el agregado pictórico.

\subsection{Participantes}

Participaron voluntariamente 147 estudiantes de grado de una universidad pública sudamericana (Edad $M=24.37, D S=8,28$ ). La muestra estuvo compuesta por 107 mujeres $(72,8 \%)$ y 40 varones $(27,2 \%)$. Los participantes fueron informados acerca del propósito del estudio y del procedimiento experimental y prestaron su consentimiento por escrito. Una vez concluido el experimento, las hipótesis y los resultados esperados fueron transmitidos grupalmente.

\subsection{Diseño y materiales}

Se utilizó un diseño experimental de un factor con tres niveles para grupos independientes. El factor fue la Modalidad de Presentación del texto, que se manipuló entre participantes. Para ello, la muestra total fue dividida aleatoriamente en tres grupos y cada grupo recibió el texto expositivo en alguna de las tres condiciones de presentación. Las condiciones fueron:

(1) texto con DS verbal (condición Solo Detalle Verbal),

(2) texto con DS verbal e ilustración asociada al mismo (condición MM Seductora), y

(3) texto con DS verbal e ilustración efectiva (condición MM Efectiva).

El texto expositivo consistió en una explicación del proceso físico-químico requerido para producir un aerogel (una sustancia liviana con propiedades cruzadas de gas y líquido). La extensión del material fue de 235 palabras, no incluyó título ni subtítulos y fue dividido en 23 líneas, presentadas en dos páginas. Cada página presentó sub-temas específicos dentro del tópico principal. La primera página (13 líneas) tuvo como objetivo introducir el tema a partir de una breve definición y descripción de los aerogeles (3 líneas) y explicar los pasos del proceso de secado utilizado para la creación de los mismos (10 líneas). En la segunda página (10 líneas) se expuso el concepto de punto crítico (niveles específicos de temperatura y presión requeridos para crear aerogeles).

En todas las condiciones, el DS fue presentado en la primera página del material y consistió en una anécdota biográfica acerca del creador de los aerogeles (i.e. "Lamentablemente, Kistler murió poco antes de que el mundo se interesase por su descubrimiento"). El uso de información biográfica como DS en textos de ciencias ha sido previamente establecido en la bibliografía (Rowland et al., 2009). Asimismo, el 
carácter seductor del detalle biográfico usado aquí respecto del resto del material y la baja familiaridad de la población con los contenidos del texto han sido establecidos y reportados previamente (Saux \& Irrazabal, 2014). El DS fue presentado siempre en la quinta línea de la primera página y representó el 6\% de la extensión total del material (medido en cantidad de palabras). No se incluyó información seductora en la segunda página, con el objeto de poder luego comparar la representación del texto como un todo (ambas páginas juntas), o en segmentos con y sin información seductora (pág. 1 vs. pág. 2).

A su vez, se utilizaron dos representaciones como material pictórico en las condiciones MM:

- La imagen seductora consistió en el retrato del creador de los aerogeles (condición MM seductora).

- La imagen efectiva consistió en un diagrama con tres nodos (cajas), representando los contenidos principales del texto (condición MM efectiva). Las cajas y las flechas fueron acompañadas de etiquetas verbales de una palabra para indicar su función dentro del diagrama.

Ambas imágenes se presentaron en forma connotativa (sin que el texto hiciese mención explícita a la imagen). Se tuvo en cuenta que la connotación sea frecuente en libros de texto de ciencias ilustrados en español (Perales \& Jiménez, 2002).

El material textual puede ser consultado en el Anexo A. Los materiales pictóricos utilizados en las condiciones MM pueden ser consultados en el Anexo B.

\subsubsection{Indicadores post-lectura}

Se utilizaron tres indicadores:

(1) Titulación: se solicitó a los participantes que seleccionasen el título que mejor representase los contenidos del texto entre tres opciones. La opción 1, considerada un Título Específico, buscó sintetizar el asunto principal del texto (i.e. Aerogeles: cómo obtenerlos). La opción 2, considerada un Título Seductor, se focalizó en el detalle biográfico, sugiriendo una organización del texto como relato histórico más que una exposición de procesos físico-químicos (i. e. Aerogeles: breve historia de su invención). De esta manera, se buscó examinar si el DS llevaba a los participantes a activar un esquema incorrecto para el texto. La 'tendencia narrativa' a simplificar las explicaciones científicas que incluyen datos históricos para tratarlas como relatos simples ha sido previamente establecida por la investigación (Carretero \& Montanero, 2008). Finalmente, la opción 3, considerada una Respuesta Ambivalente, afirmaba que ambos títulos eran igualmente buenos. El uso de la titulación como indicador de la comprensión textual, y en particular de la activación de esquemas de nivel 
superior que permiten identificar el asunto central del material, ha recibido apoyo dentro de la psicología del discurso (León \& Martin, 1993; Maturano, Mazitelli \& Macias, 2010). Sin embargo, no ha sido una variable frecuente en las investigaciones sobre DS.

(2) Recuerdo libre: se solicitó escribir la información recordada del texto (7 líneas máx.). El propósito de la tarea de recuerdo fue examinar la retención de las ideas contenidas en el texto.

(3) Verificación literal e inferencial de afirmaciones basadas en el texto: se solicitó responder si 16 afirmaciones relativas al texto eran verdaderas, falsas, o si se desconocía la respuesta. La mitad de las afirmaciones se basó en contenido de la primera página del texto, la otra mitad se basó en contenidos de la segunda página. Adicionalmente, la mitad fueron afirmaciones literales (idénticas a oraciones del texto), y la otra mitad fueron afirmaciones inferidas (información que puede derivarse del texto aunque no esté mencionada explícitamente). De este modo, los participantes debieron contestar cuatro afirmaciones literales y cuatro afirmaciones inferidas para cada página del texto. El orden de presentación de las afirmaciones en el cuadernillo, así como el orden de presentación de las opciones de respuesta, fue aleatorizado dentro de cada condición.

Adicionalmente, con el objeto de indagar la familiaridad con los contenidos, se solicitó a los participantes que indicasen en una escala con valores entre 1 ('nada') y 9 ('todo') su grado de conocimiento sobre los contenidos del texto.

\subsection{Procedimiento}

El estudio se dividió en dos fases. La primera fase consistió en la lectura del material experimental en papel, en alguna de las tres condiciones. La segunda fase consistió en el completamiento de los indicadores de recuerdo y comprensión en un cuadernillo. Esta segunda fase fue dividida, a su vez, en dos momentos: sin disponibilidad del texto (cuadernillo A) y con disponibilidad del texto (cuadernillo B). El cuadernillo A incluyó las actividades de titulación y de recuerdo libre. El cuadernillo B incluyó la tarea de verificación y la escala de familiaridad con el tema. Los cuadernillos siempre se administraron en orden A-B, para evitar efectos causados por la re-lectura. La toma fue realizada en sesiones de grupos de 30 participantes aproximadamente. La duración total de la toma fue de 25 minutos.

\section{Resultados}

\subsection{Familiaridad con el tema}

En primer lugar, se analizó la escala incluida en el cuadernillo B. El texto recibió puntajes bajos en familiaridad, tanto en la condición de Solo Texto $(M=1.6, D S=1.5)$, 
como en las condiciones MM Seductora $(M=2.07$, $D S=1.6)$ y MM Efectiva $(M=$ 2.08, $D S=1.8$ ). A continuación, se analizó el efecto de la Modalidad de Presentación del texto sobre los puntajes de Familiaridad, mediante un análisis de varianza de un factor para grupos independientes. El efecto de la Modalidad de Presentación no fue significativo, $F(2,143)=.92, M S_{e}=2.94, p=.39$.

\subsection{Selección de Título}

Se realizó una prueba de independencia de Chi Cuadrado sobre las frecuencias de la Selección de Título discriminadas por condición. Se halló una asociación significativa entre la Selección del Título y la Modalidad de Presentación del texto, $X^{2}(4, N=145)$ $=16.6, p=.002$. El análisis de los residuos estandarizados reveló que, en la condición MM Seductora, un número significativamente mayor de sujetos eligió el Título Seductor $(64,29 \%, Z=1.9, p<.05)$, mientras que menos sujetos eligieron el Título Ambivalente $(7,14 \%, Z=-2,7, p<.05)$. No se hallaron asociaciones significativas para la Selección de Título en el resto de las modalidades de presentación del texto. La Tabla 1 muestra la frecuencia y los residuos tipificados de cada combinación de título seleccionado y la Modalidad de Presentación del texto.

Tabla 1. Porcentajes y residuos tipificados para el Título Seleccionado según la Presentación del Texto.

\begin{tabular}{|c|c|c|c|c|c|}
\hline & & & \multicolumn{3}{|c|}{ Titulo Seleccionado } \\
\hline & & & Específico & Seductor & Ambivalente \\
\hline \multirow{6}{*}{$\begin{array}{l}\text { Presentación } \\
\text { del texto }\end{array}$} & Solo Detalle Verbal & \% de Condición & $20.0 \%$ & $43.3 \%$ & $36.7 \%$ \\
\hline & & Res. tipificados & -.7 & -.4 & 1.3 \\
\hline & MM Seductora & \% de Condición & $28.6 \%$ & $64.3 \%$ & $7.1 \%$ \\
\hline & & Res. tipificados & .3 & $1.9^{a}$ & $-2.7^{a}$ \\
\hline & MM Efectiva & $\%$ de Condición & $27.1 \%$ & $37.3 \%$ & $35.6 \%$ \\
\hline & & Res. tipificados & .1 & -1.3 & 1.7 \\
\hline \multicolumn{2}{|l|}{ Total } & & $26.2 \%$ & $49.0 \%$ & $24.8 \%$ \\
\hline
\end{tabular}

Nota. Frecuencia expresada en porcentajes.

a Valor critico para los residuos: $Z=+/-1.96(\alpha=.05)$. Las celdas con valores por encima o por debajo del valor crítico se encuentran resaltadas.

\subsection{Recuerdo del texto}

Para poder analizar los protocolos de recuerdo, se dividió el texto en tres segmentos y se identificaron las ideas contenidas en cada segmento. En el primer segmento (tres primeras líneas de la primera página), se identificó una idea. En el segundo segmento (10 líneas restantes de la primera página) se identificaron 6 ideas. En el tercer segmento (10 líneas de la segunda página) se identificaron cinco ideas. Se asignó 
un punto por cada idea presente en el protocolo y 0 por cada idea no mencionada. En general, el recuerdo del texto fue bajo para las tres condiciones (3 a 4 puntos sobre un puntaje máximo de 12). Se realizaron ANOVAs de una vía sobre la cantidad de ideas recordadas, tomando la Modalidad del Texto como factor. Los análisis no mostraron efectos significativos para el recuerdo total del texto, $F(2,144)=2.206$, $M S_{e}=3.054, p=.114$. Sin embargo, el análisis por página y por segmento reveló un efecto significativo de la Modalidad en el recuerdo de la primera página, en particular del segundo segmento, $F(2,144)=5.563, M S_{e}=1.746, p=.005$. Comparaciones posthoc pareadas (HSD de Tukey) revelaron que el grupo de la condición MM Efectiva recordó una cantidad significativamente mayor de ideas del segundo segmento de la primera página del texto $(M=2.3, D S=1.4)$, en comparación con las condiciones MM Seductora $(M=1.6, D S=1.1, p=.008)$ y Solo Detalle Verbal $(\mathrm{M}=1.6, \mathrm{DS}=$ $1.2, \mathrm{p}=.036)$. Ningún otro contraste mostró diferencias significativas.

\subsection{Verificación de afirmaciones}

Se analizaron las respuestas a la actividad de verificación de frases teniendo en cuenta el carácter literal o inferido de la afirmación, así como si la misma había sido formulada sobre la base de los contenidos de la primera o la segunda página del texto. Se procedió a calcular la cantidad de errores sobre las cuatro afirmaciones literales y las cuatro afirmaciones inferidas para cada página. En líneas generales, la tasa de errores fue baja en todas las condiciones, especialmente en la verificación de afirmaciones literales. No obstante, se encontró un efecto significativo de la Modalidad del Texto sobre la cantidad de errores en la verificación de frases de la primera página del texto, $F(2,142)=4.109, M S_{e}=.502, p=.018$. El análisis por tipo de error reveló un efecto solo para las afirmaciones inferidas de la primera páginam $F$ $(2,141)=3.614, M S_{e}=.307, p=.029$. Las pruebas post-hoc (HSD de Tukey) mostraron que la condición MM Efectiva tuvo una cantidad significativamente menor de errores en la verificación de afirmaciones inferidas $(M=.31, D S=.4)$, en comparación con la condición MM Seductora $(M=.58, D S=.6, p=.02)$, pero similar a la condición Solo Detalle Verbal $(M=.4, D S=.5, p=.58)$. Ninguna otra comparación mostró diferencias significativas.

\section{Discusión}

\subsection{Contribuciones empíricas}

Los resultados permiten sostener que la presentación multimedia influyó sobre el procesamiento del material textual, y que el uso estratégico de las representaciones pictóricas modula el efecto de dicha influencia. Esta afirmación se basa en que las condiciones multimedia se diferenciaron en sentidos opuestos respecto de la condición Solo Detalle Verbal. 
En relación con la primera hipótesis específica (mediciones descendidas para el grupo que leyó el texto ilustrado con el objeto de resaltar el DS) se observa que, en efecto, los participantes que vieron el detalle en versión multimedia eligieron el título seductor con más frecuencia, recordaron menos ideas y presentaron más errores en la verificación de enunciados que el resto de los participantes. Puede afirmarse entonces que el apuntalamiento pictórico de un DS representa la situación de mayor perjuicio para el procesamiento cognitivo del texto, incluso en comparación con condiciones seductoras exclusivamente verbales. Estos resultados son consistentes con la investigación previa sobre el uso estratégico de las imágenes en los textos educacionales (Sánchez \& Wiley, 2006; Mayer et al., 2008; Sung \& Mayer, 2012). Asimismo, consideramos que aportan al corpus actual de datos de dos maneras. En primer término, replican el efecto seductor y el efecto multimedia en un grupo de estudiantes universitarios hispanoparlantes, una población con poca representación en el área. En segundo término, mientras que los estudios previos utilizan la imagen irrelevante como un elemento seductor en sí mismo, la imagen incluida en la condición MM Seductora (retrato en niveles de gris) no fue considerada un elemento atractivo per se, sino un señalador pictórico de contenidos seductores escritos (el detalle biográfico). Recientemente, Sung y Mayer (2012) han propuesto tres tipos de imágenes usadas en presentaciones multimedia con fines educacionales, que varían en función de su relevancia e interés y que producen distintos efectos sobre la elaboración mental del texto: las imágenes efectivas (gráficos relevantes para el objetivo educativo y variables en interés), las imágenes seductoras (muy interesantes pero irrelevantes en términos educativos) y las imágenes decorativas (neutras e irrelevantes). Atendiendo a esta distinción, el tipo de imagen utilizada en la condición MM Seductora conformaría un caso poco contemplado por la investigación previa, aunque acaso frecuente en los textos de ciencias educativos: imágenes intrínsecamente neutras (decorativas) que cumplen un rol seductor en tanto se hallan connotadas por información verbal seductora (Schraw, 1998). En este sentido, este trabajo aporta al estudio de los diferentes tipos de representaciones pictóricas y su vínculo con el fenómeno DS.

En relación con la segunda hipótesis específica (reducción del efecto seductor en el grupo que recibió el texto con un gráfico efectivo), se observa que los participantes en la condición MM Efectiva recordaron más ideas que las otras dos condiciones (medida de recuerdo), pero no se diferenciaron de la condición Solo Detalle Verbal en la selección del título, ni en la cantidad de errores en la verificación de frases (medidas de comprensión). En consistencia con hallazgos previos (Rowland et al., 2009), esto podría interpretarse como evidencia de que los gráficos diseñados para promover la comprensión del mensaje son capaces de mitigar el efecto de los DS verbales, particularmente en actividades asociadas al recuerdo del texto. Sin dudas, consideramos que esta última afirmación requiere de mayor indagación, en tanto indicaría que la señalización pictórica de los contenidos esenciales de un texto que contiene a su vez DS verbales puede resultar útil para potenciar el recuerdo de los contenidos, pero no para promover la integración inferencial de los mismos. 


\subsection{Contribuciones teóricas}

Como se expuso en el marco teórico, la TCAM ha propuesto diversas hipótesis para explicar el modo en los que los DS afectan la actividad cognitiva (alteración durante la selección, alteración durante la organización y alteración durante la integración, Harp \& Mayer, 1998; Mayer et al., 2008). Mientras que la alteración de la integración predice dificultades en la elaboración del asunto central del texto (debido a la desviación hacia esquemas inapropiados), la explicación atencional predice dificultades focalizadas en la representación de los segmentos textuales próximos a la información seductora (debido a la alteración de la selección y la organización durante la lectura).

Diferentes estudios han provisto evidencia empírica consistente con alguna de estas hipótesis e inconsistente con las demás (Rey, 2012). Como resultado, si bien coexisten varias explicaciones, no hay acuerdo teórico acerca de si los DS afectan un proceso cognitivo en particular o pueden influir sobre diferentes mecanismos. Debido al uso de tareas con y sin disponibilidad del texto, así como de indicadores de recuerdo y de integración inferencial macro y micro estructurales (titulación y preguntas acotadas a segmentos del texto, respectivamente), los resultados obtenidos contribuyen a esta discusión teórica, sugiriendo que ninguna de las hipótesis explicaría completamente el efecto de los DS. Esta afirmación se apoya en que el grupo que vio el DS en versión multimedia mostró un desempeño empobrecido: a) tanto en tareas sin disponibilidad del texto (recuerdo y titulación), como en la tarea que permitió la revisión del texto durante su completamiento (preguntas inferenciales); y b) tanto en el indicador de la comprensión del asunto central (selección del título), como en los indicadores del procesamiento de contenidos puntuales (recuerdo y verificación de la página que contenía el DS).

Consideramos que estos resultados indican la naturaleza múltiple del llamado efecto seductor, señalando la importancia para la investigación de examinar la influencia conjunta sobre los procesos atencionales y de esquematización, en lugar de intentar aislar una sola hipótesis.

\subsection{Limitaciones}

En primer lugar, si bien la investigación previa ha reportado una continuidad en los resultados empíricos hallados en los formatos impreso y electrónico (Mayer, 2008), este estudio presentó el material en papel, por lo que las generalizaciones de los resultados no deberían extenderse sin estudios adicionales a presentaciones en pantalla. En segundo lugar, es importante destacar que los resultados aquí reportados se circunscriben a la primera lectura por parte de estudiantes universitarios de textos con fines educativos, relativamente breves y que presentan explicaciones de mecanismos físico-químicos, no debiendo extrapolarse directamente a todo tipo de texto. Asimismo, el uso de información biográfica como DS debería ser extendido con cautela a otros contextos, como el estudio histórico o el análisis de las fuentes, en 
los cuales los datos biográficos no son irrelevantes para los procesos de comprensión textual (Perfetti et al., 1999). En tercer lugar, debe tenerse en cuenta que los resultados y conclusiones teóricas derivadas de este estudio solo deberían generalizarse a lectores con bajo conocimiento previo en el tema. Desde la psicolingüística del texto, se ha señalado que la alta coherencia de un texto (en el sentido en que explicita muchas de las relaciones entre sus ideas) no siempre es mejor. En el caso de lectores expertos, un grado elevado de coherencia textual puede llevar a un procesamiento superficial (McNamara, Kintsch, Butler-Songer \& Kintsch, 1996). Por lo tanto, es posible que las manipulaciones realizadas produjesen efectos diferentes a los aquí reportados en una muestra de lectores expertos. En cuarto lugar, consideramos que las mediciones de memoria e integración inferencial posteriores a la lectura utilizada en este estudio se beneficiarían de la complementación con datos on-line, como mediciones oculométricas, que reflejen los procesos atencionales selectivos durante el rastreo visual de la presentación multimedia. Finalmente, consideramos importante señalar que el control previo del interés de los materiales utilizados (reportado en Saux \& Irrazabal, 2014) fue realizado sobre la base de una escala de interés unidimensional (“Cuán interesante te resulta esta idea?"). Si bien este control sigue la práctica común en el área (Mayer et al., 2008), podría estar haciendo un uso acaso simplista del concepto teórico de 'interés', descripto como un fenómeno complejo y multidimensional desde la psicología de la motivación (Hidi, Renninger \& Krapp, 2004). Consideramos que esta limitación no se circunscribe a este estudio y se extiende a la investigación multimedia en general, que ha tendido a poner el foco en el procesamiento de la información más que en el afecto despertado por los materiales (Mayer et al., 2008). Sin embargo, la TCAM ha mostrado recientemente un interés por incorporar nociones motivacionales (Mayer, 2014), por lo que consideramos que los presentes resultados deberían ser complementados con estudios orientados a examinar con mayor detalle el aspecto emocional y/o interés despertado por los materiales.

\section{CONCLUSIONES}

Los resultados del presente trabajo son consistentes con la TCAM, que sostiene que los agregados pictóricos pueden conllevar tanto costos como beneficios cognitivos a la tarea de comprender un texto de ciencias. El foco de esta investigación estuvo puesto en el uso estratégico de estos agregados, en tanto se asume que la clave para producir uno u otro efecto radica en el mejor entendimiento de la interacción de los procesos psicológicos del lector con las características del mensaje presentado.

Consideramos que la facilitación actual en el acceso a la información científica comporta varios desafíos para el lector con bajo conocimiento previo. Por un lado, existe la ventaja de llegar rápida y fácilmente a mucha información. Por otro lado, esta situación presenta al lector novato el problema de distribuir estratégicamente sus capacidades de procesamiento frente al aumento de material escrito a su disposición, en el cual se puede recurrir al uso de información tangencial pero interesante 
para capturar su interés. Siguiendo lo propuesto por Britt, Richter y Rouet (2014), consideramos que estos desafíos asociados a la era de la información no son nuevos en esencia. En particular, en el caso de los mensajes multimedia, el estudio de las presentaciones conjuntas de texto e imagen cuenta ya con una tradición en la psicología cognitiva que comienza a sistematizarse a comienzos de la década del ' 80 (Levie \& Lentz, 1982). Sin embargo, la novedad radica en que estos desafíos se han extendido de forma vertiginosa a los aprendices con bajo conocimiento previo, que se ven expuestos a la sobreabundancia informativa por fuera del dispositivo educacional formal, en donde la figura del enseñante suele delinear indicaciones generales o circunscribir la lectura a ciertos objetivos.

En términos de la investigación, creemos que esta situación requiere profundizar en el estudio de las características objetivas de los mensajes escritos que puedan facilitar la tarea al lector lego, acompañando los mencionados cambios en los contextos de lectura. En este sentido, los resultados obtenidos indican la importancia de atender a la forma en que se diseña y planea la inclusión de imágenes en los textos, teniendo en cuenta no solo criterios formales, editoriales o publicitarios, sino también psicológicos.

En suma, el estudio de los procesos implicados en la comprensión de las exposiciones científicas acompañadas de imágenes por parte de lectores con bajo conocimiento previo constituye un desafío actual, cada vez más urgente. Asimismo, a medida que las nuevas tecnologías se expanden, investigar la comprensión a partir de materiales en múltiples formatos representacionales será esencial para poder asociar los cambios en los contextos sociales con beneficios significativos en el nivel cognitivo. 


\section{REFERENCIAS BIBLIOGRÁFICAS}

Amadieu, F. \& Salmerón, L. (2014). Concept maps for comprehension and navigation of hypertexts. En R. Hanewald \& D. Ifenthaler (Eds.), Digital Knowledge Maps in Education (pp. 41-59). Nueva York: Springer.

Bassols, M. \& Torrent, A. (1997). Modelos textuales. Barcelona: Octaedro.

Britt, M. A., Richter, T. \& Rouet, J. F. (2014). Scientific Literacy: The role of goaldirected reading and evaluation in understanding scientific information. Educational Psychologist, 49, 104-122.

Carretero, M. \& Montanero, M. (2008). Enseñanza y aprendizaje de la historia: Aspectos cognitivos y culturales. Cultura y Educación, 20(2), 133-142.

Chung, M., Oden, R. P., Joyner, B. L., Sims, A. \& Moon, R.Y. (2012). Safe infant sleep recommendations on the Internet: Let's Google it. The Journal of Pediatrics, 161, 1080-1084.

de Vega, M. \& Cuetos, F. (1999). Psicolingüistica del español. Madrid: Trotta.

de Vega, M., Glenberg, A. \& Graesser, A. C. (2008). Symbols, embodiment, and meaning. Nueva York: Oxford University Press.

Escudero, I. \& León, J. A. (2007). Procesos inferenciales en la comprensión del discurso escrito. Influencia de la estructura del texto en los procesos de comprensión. Revista Signos. Estudios de Lingüistica, 40(64), 311-336.

Garner, R. (1990). When children and adults do not use learning strategies: Toward a theory of settings. Review of Educational Research, 60, 517-529.

Garner, R., Gillingham, M. G. \& White, C. S. (1989). Effects of seductive details on macroprocessing and microprocessing in adults and children. Cognition and Instruction, 6, 41-57.

Graesser, A., León, J. A. \& Otero J. (2002). Introduction to the psychology of science text comprehension. En J. Otero, J. A. León \& A. Graesser (Eds.). The Psychology of science text comprehension (pp. 1- 15). Mahwah, N.J.: Erlbaum.

Gyselinck, V. \& Tardieu, H. (1999). The role of illustrations in text comprehension. En H. van Oostendorp \& S. R. Goldman (Eds.), The construction of mental representations during reading (pp. 195-218). N. J.: Erlbaum.

Harp, S. F. \& Mayer, R. E. (1997). The role of interest in learning from scientific text and illustrations: On the distinction between emotional and cognitive interest. Journal of Educational Psychology, 89, 92-102. 
Harp, S. F. \& Mayer, R. E. (1998). How seductive details do their damage: A theory of cognitive interest in science learning. Journal of Educational Psychology, 90, 414-434.

Harp, S. F. \& Maslich, A. A. (2005). The consequences of including seductive details during lecture. Teaching of Psychology, 32(2), 100-103.

Hidi, S. \& Renninger, K. A. (2006). The four-phase model of interest development. Educational Psychologist, 41(2), 111-127.

Hidi, S., Renninger, K. A. \& Krapp, A. (2004). Interest, a motivational construct that combines affective and cognitive functioning. En D. Y. Dai \& R. J. Sternberg (Eds.), Motivation, emotion and cognition (pp.89-115). Mahwah, N.J.: Erlbaum.

Jaeger, A. J. \& Wiley, J. (2014). Do illustrations help or harm metacomprehension accuracy? Learning and Instruction, 34, 58-73.

Just, M. A. \& Carpenter, P. A. (1992). A capacity theory of comprehension: Individual differences in working memory. Psychological Review, 1, 122-149.

Kintsch, W. (1998). Comprehension: A paradigm for cognition. Nueva York: Cambridge University Press.

Kintsch, W. \& van Dijk, T. A. (1978). Towards a model of text comprehension and production. Psychological Review, 85, 363-394.

Kress, G. \& Van Leeuwen, T. (2001). Multimodal discourse. The modes and media of contemporary communication. Londres: Arnold.

Lehman, S., Schraw, G., McCrudden, M. T. \& Hartley, K. (2007). Processing and recall of seductive details in scientific text. Contemporary Educational Psychology, 32, 569-587.

León, J. A. \& Martín A. (1993). El título como recurso didáctico. Comunicación, Lenguaje y Educación, 19, 159-170.

Levie, W. H. \& Lentz, R. (1982). Effects of text illustrations: A review of research. Educational Communication and Technology Journal, 30, 195-232.

Levin, J. \& Mayer, R. (1993). Understanding illustrations in text. En B. K. Britton Woodward \& M. Brinkley (Eds.), Learning from Textbooks (pp. 95-113). N. J.: Erlbaum.

Maturano, C., Mazitelli, C. \& Macías, A. (2010). Detección de dificultades básicas de estudiantes de escuela secundaria en la comprensión de un texto de Física. Latin-American Journal of Physics Education, 4(1), 160-167. 
Mayer, R. (2001). Multimedia learning. Nueva York: Cambridge University Press.

Mayer, R. (2005). Cambridge handbook of multimedia learning. Nueva York: Cambridge University Press.

Mayer, R. (2008). Applying the science of learning: Evidence-based principles for the design of multimedia instruction. American Psychologist, 63(8), 760-769.

Mayer, R. (2009). The next phase in multimedia learning. En S. Kalyuga (Ed.) Managing cognitive load in adaptive multimedia learning (pp. 10-12). Nueva York: Information Science Reference.

Mayer, R. (2010). Applying the science of learning to medical education. Medical Education, 44(6), 543-549.

Mayer, R. (2014). Incorporating motivation into multimedia learning. Learning and Instruction, 29, 171-173.

Mayer, R., Heiser, J. \& Lonn, S. (2001). Cognitive constraints on multimedia learning: When presenting more material results in less understanding. Journal of Educational Psychology, 93, 187-198.

Mayer, R., Griffith, E., Jurkowitz, I. \& Rothman, D. (2008) Increased interestingness of extraneous details in a multimedia science presentation leads to decreased learning. Journal of Experimental Psychology: Applied, 14, 329-339.

Meyer, B. (1985). Prose analysis: Purposes, procedures, and problems. En B. K. Britton \& J. Black (Eds.), Analyzing and understanding expository text, (pp. 269304). Hillsdale, N. J.: Erlbaum.

McCrudden, M. T. \& Corkill, A. J. (2010). Verbal ability and the processing of scientific text with seductive detail sentences. Reading Psychology, 31(3), 282-300.

McNamara, D. (2004). Aprender del texto: Efectos de la estructura textual y las estrategias del lector. Revista Signos. Estudios de Lingüística, 37(55), 19-30.

McNamara, D. S., Kintsch, E., Butler-Songer, N. \& Kintsch, W. (1996). Are good texts always better? Interactions of text coherence, background knowledge, and levels of understanding in learning from text. Cognition and Instruction, 14(1), 1-43.

Molinari Marotto, C. (2005). La generación de inferencias emocionales en la comprensión de narraciones: Evidencia experimental e implementación en el modelo computacional Landscape. Tesis doctoral inédita, Universidad de Buenos Aires, Argentina.

Moreno, R. \& Mayer, R. (1999). Cognitive principles of multimedia learning: The role of modality and contiguity. Journal of Educational Psychology, 91, 358-368. 
Padilla, C., Douglas, S. \& López, E. (2007). Yo expongo. Taller de prácticas de comprensión y producción de textos expositivos. Córdoba: Comunicarte.

Park, B., Moreno, R., Seufert, T. \& Brünken, R. (2011). Does cognitive load moderate the seductive details effect? A multimedia study. Computers in Human Behavior, $27,5-10$.

Parodi, G. (2010). Multisemiosis y lingüística de corpus: Artefactos (multi) semióticos en los textos de seis disciplinas en el corpus PUCV-2010. Revista de Lingüística Teórica y Aplicada, 48(2), 33-70.

Perales, F. \& Jiménez, J. (2002). Las ilustraciones en la enseñanza-aprendizaje de las ciencias. Análisis de libros de texto. Enseñanza de las ciencias, 20, 369-386.

Perales, F. (2006). Uso (y abuso) de la imagen en la enseñanza de las ciencias. Enseñanz̧a de las Ciencias, 24(1), 13-30.

Perfetti, C. A., Rouet, J.-F. \& Britt, M. A. (1999). Towards a theory of documents representation. En H. van Oostendorp \& S. R. Goldman (Eds.), The construction of mental representations during reading (pp. 99-122). Mahwah, N.J.: Erlbaum.

Rey, G. D. (2012). A review of research and a meta-analysis of the seductive detail effect. Educational Research Review, 7, 216-237.

Rouet, J.-F. \& Britt, M. A. (2011). Relevance processes in multiple document comprehension. En M.T. McCrudden, J. P. Magliano \& G. Schraw (Eds.), Relevance Instructions and Goal-focusing in Text Learning (pp. 19-52). Greenwich, CT: Information Age.

Rowland, E., Skinner, C. H., Davis-Richards, K., Saudargas, R. \& Robinson, D. H. (2008). An investigation of placement and type of seductive details: The primacy effect of seductive details on text recall. Research in the Schools, 15(2), 80-90.

Rowland, E., Skinner, C. H., Skinner, A. L., Saudargas, R., Robinson, D. H. \& Kirk, E. R. (2009). Investigating the interaction of graphic organizers and seductive details. Research in the Schools, 16(2), 29-40.

Sánchez, C. \& Wiley, J. (2006). Effects of working memory capacity on learning from illustrated text. Memory \& Cognition, 34(2), 344-355.

Salmeron, L., Canas, J., Kintsch, W. \& Fajardo, I. (2005). Reading strategies and hypertext comprehension. Discourse Processes, 40(3), 71-91

Saux, G. \& Irrazabal, N. (2014). El uso de detalles biográficos como información seductora en el texto de ciencias. Revista de Psicología, 19, 45-51. 
Schnotz, W. (2005). An integrated model of text and picture comprehension. En R. Mayer (Ed.), Cambridge Handbook of Multimedia Learning (pp. 49-69). Cambridge: Cambridge University Press.

Schraw, G. (1998). Processing and recall differences among seductive details. Journal of Educational Psychology, 90, 3-12.

Sung, E. \& Mayer, R. (2012). When graphics improve liking but not learning from online lessons. Computers in Human Behavior, 28, 1618-1625.

van Dijk, T. A. \& Kintsch, W. (1983). Strategies of discourse comprehension. Nueva York: Academic Press.

Wade, S. E., Schraw, G., Buxton, W. M. \& Hayes, M. T. (1993). Seduction of the strategic reader: Effects of interest on strategies and recall. Reading Research Quarterly, 28, 3-24.

Wiley, J., Ash, I. K., Sánchez, C.A. \& Jaeger, A. (2011). Clarifying readers' goals for learning from expository science texts. En M. McCrudden, J. Magliano \& G. Schraw (Eds.), Text relevance and learning from text (pp. 353-374). Greenwich, CT: Information Age Publishing. 


\section{ANEXO}

Material textual utilizado en las tres condiciones experimentales

Pág. 1, segm. 1

Los aerogeles son, hoy por hoy, uno de los materiales menos densos y más livianos que se conocen. Deben su liviandad a una gran porosidad. Alrededor del 95\% del volumen total de un aerogel está compuesto por espacios llenos de aire.

Pág. 1,

segm. 2

El primer aerogel fue creado en 1931 por el ingeniero químico S. Kistler, quien, lamentablemente, murió poco antes de que el mundo se interesarse por su descubrimiento*. Los aerogeles son hechos a partir de un proceso conocido como "secado supercrítico". El secado supercrítico comienza con un gel común, normalmente de sílice. El gel común se licúa con gas, logrando extraer la porción líquida del gel. El fluido resultante es secado con un proceso especial. El proceso lleva la presión y temperatura del fluido a un nivel específico, llamado "nivel crítico". La sustancia resultante es el aerogel.

Pág. 2,

$\operatorname{segm} 3$

El "nivel crítico" de una sustancia separa sus fases líquida y gasesosa. Una sustancia que alcanza su nivel crítico presenta características cruzadas entre las de un líquido y las de un gas. El secado supercrítico permite alcanzar este nivel. Casi todo el líquido del fluido original es reemplazado por poros de aire, sin que se pierda la estructura en el material resultante. Muchas son las aplicaciones de los aerogeles hoy en día. En un futuro cercano, seguramente habrá muchos más avances en la tecnología de los aerogeles.

* DS resaltado en cursiva y en negrita (sin resaltado en el original)
Imagen utilizada en las condición MM Seductora

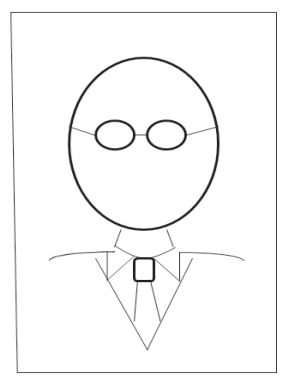

S. Kistler, creador de los aerogeles**

** Ejemplo del tipo de imagen incluida (imagen original sujeta a derechos de autor).

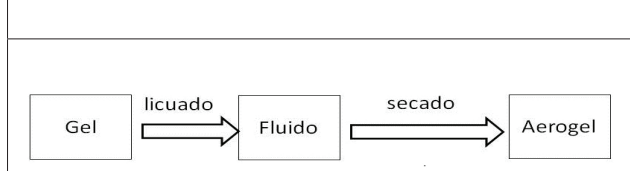

Pasos en el proceso de secado supercrítico 